\title{
Oferta e utilização de teleconsultorias para Atenção Primária à Saúde no Programa Telessaúde Brasil Redes
}

\author{
Provision and use of teleconsultations for Primary Health Care in the Telehealth Brazil \\ Networks Program
}

\author{
Oferta y uso de teleconsultorías para Atención Primaria de Salud en el Programa Telesalud \\ Brasil Redes \\ Carlos André Aita Schmitza , Erno Harzheim ${ }^{b}$
}

\section{Resumo}

Objetivo: Avaliar a adequação do Programa Telessaúde Brasil Redes, no âmbito das teleconsultorias, a partir da análise de dados de estrutura e processo da Plataforma Nacional de Telessaúde. Método: Estudo descritivo, com análise estatística de série histórica (24 meses) da produção de teleconsultoria pelos núcleos de telessaúde que utilizaram a ferramenta de outubro de 2013 a setembro de 2015. Resultados: Foram respondidas 29.575 teleconsultorias por 18 núcleos de telessaúde em todo o país, para 43.421 usuários em 9.801 unidades de saúde. Oferta e demanda mensal variaram entre 0,92 a 2,06 e 0,22 a 1,00 teleconsultorias, respectivamente. O percentual de unidades de saúde com ao menos uma solicitação/mês manteve-se próximo a $0,1 \%$, sendo que $87,3 \%$ dos usuários cadastrados não realizou nenhuma solicitação no período. Os temas solicitados cobriram todos os capítulos da Classificação Internacional da Atenção Primária e da Classificação Internacional de Doenças. A satisfação dos usuários foi de 95,6\% e o percentual de dúvidas totalmente respondidas foi $88,4 \%$. Conclusões: Apesar da oferta adequada em relação às metas do programa, a demanda é muito baixa, gerando capacidade instalada ociosa de um grupo de teleconsultores com capacidade de resposta para um amplo leque de temas. Levanta-se a necessidade de ganho de escala, integração horizontal e fortalecimento da telerregulação e da auditoria das ações de telessaúde, com centralização de recursos e redução do número de núcleos de telessaúde. Além disso, é necessário investir em novas tipologias sinérgicas e sistêmicas de oferta de ações de telessaúde, como o apoio ao complexo regulador ambulatorial e à orientação da população.
Palavras-chave:

Atenção Primária à Saúde

Telemedicina

Avaliação de Programas

e Projetos de Saúde

Informática Médica

\begin{abstract}
Objective: To evaluate the adjustment of teleconsultations provided by a large telehealth program in Brazil (Telehealth Brazil Networks) based on the analysis of National Telehealth Platform structure and process data. Method: This descriptive study involves statistical analysis of a historical series (24 months) of teleconsultations provided by 18 telehealth groups across the country between October 2013 and September 2015. Results: During the study period, 29,575 teleconsultations were provided by 18 telehealth groups to 43,421 users from 9,801 health units. The average number of teleconsultations per consultant per month ranged from 0.92 to 2.06 , and the average number of requests per user per month ranged from 0.22 to 1.00 . The percentage of health units with at least one request/month remained close to $0.1 \%$ throughout the study period, with no request at all from $87.3 \%$ of the users during this time. The requests for teleconsultations covered all the chapters of the International Ranking of Primary Care and of the International Ranking of Diseases. User satisfaction reached $95.6 \%$, and $88.4 \%$ of the requests were fully resolved. Conclusions: Despite the adequate provision of telehealth support in relation to the goals of the Brazil Telehealth Networks program, demand is still low. As a consequence, the system remains largely idle, despite its capacity to provide answers for a broad range of subjects. This suggests the need to scale up reach, with horizontal integration and strengthening of telehealth regulation and audit steps, as well as to concentrate resources and reduce the number of telehealth groups. In addition, investments are needed to develop new types of synergistic and systemic telehealth actions, including support to the outpatient regulation system and population guidance.
\end{abstract}

Como citar: Schmitz CAA, Harzheim E. Oferta e utilização de teleconsultorias para Atenção Primária à Saúde no Programa Telessaúde Brasil Redes. Rev Bras Med Fam Comunidade. 2017;12(39):1-11. http://dx.doi.org/10.5712/rbmfc12(39)1453

\section{Keywords:}

Primary Health Care

Telemedicine

Program Evaluation

Medical Informatics

Fonte de financiamento: declaram não haver. Parecer CEP: não se aplica. Conflito de interesses: declaram não haver. Procedência e revisão por pares: revisado por pares. Recebido em: 25/01/2017. Aprovado em: 29/05/2017. 


\section{Resumen}

Objetivo: Evaluar la adecuación del Programa Telesalud Brasil Redes, en el ámbito de las teleconsultorías, a partir del análisis de datos de la estructura y proceso de la Plataforma Nacional de Telesalud. Método: Estudio descriptivo. Incluye el análisis estadístico de una serie histórica (24 meses) de teleconsultorías realizadas por 18 núcleos de telesalud en todo el país entre octubre de 2013 y septiembre de 2015. Resultados: Los 18 núcleos de telesalud realizaran 18.575 teleconsultorías para 43.421 usuarios de 9.801 unidades de salud del país. El promedio mensual de teleconsultorías por consultor osciló entre 0,92 y 2,06. Ya el promedio mensual de solicitudes por usuario osciló entre 0,22 y 1,00. El porcentaje de unidades de salud con por lo menos una solicitud cada mes se mantuvo en alrededor del $0,1 \%$ a lo largo del período de la investigación y $87,3 \%$ de los usuarios registrados no ha hecho ninguna solicitud durante el período. Los asuntos demandados en las teleconsultorías abarcaron todos los capítulos de la Clasificación Internacional de Atención Primaria y de la Clasificación Internacional de Enfermedades. La satisfacción del usuario alcanzó el 95,6\% y el $88,4 \%$ de las solicitudes se resolvieron por completo. Conclusiones: La oferta de servicios de telesalud en Brasil es adecuada. Sin embargo, la demanda aún es muy baja. Como consecuencia, el sistema permanece ocioso en gran medida, a pesar de su capacidad para responder a una amplia gama de cuestiones. Esto sugiere la necesidad de ampliar el alcance, con la integración horizontal y el fortalecimiento de la regulación de la telesalud y las medidas de auditoría, así como concentrar recursos y reducir el número de núcleos de telesalud en el pais. Además, se necesitan inversiones para desarrollar nuevos tipos de acciones sinérgicas y sistémicas de telesalud, incluyendo el apoyo al sistema de regulación ambulatoria y el apoyo a la orientación de la población.
Palabras clave:

Atención Primaria de Salud

Telemedicina

Evaluación de Programas y Proyectos de Salud Informática Médica

\section{Introdução}

A Atenção Primária à Saúde (APS) foi descrita, primordialmente em 1920, num contexto de Redes de Atenção à Saúde (RAS) ${ }^{1}$ e conceituada, contemporaneamente, como o nível preferencial de acesso para a atenção à saúde, responsável por um atendimento integral, longitudinal e coordenado. ${ }^{2}$ Seu papel de ordenadora e de centro de comunicação das RAS, definido no marco legal brasileiro, ${ }^{3}$ não tem sido atingido. Sua materialização no país, a Estratégia de Saúde da Família (ESF), apesar de cobrir $63,4 \%$ da população com suas 39.942 equipes, em outubro de 2015 , e ter alcançado vários resultados positivos, ${ }^{4-6}$ apresenta grandes heterogeneidades em termos de acesso, qualidade e custo da atenção à saúde. A fonte dessa heterogeneidade tem como causas, entre outras, deficiências na formação profissional, déficits estruturais e baixa incorporação tecnológica. ${ }^{7-10}$

A situação se repete na atenção secundária, com o agravante da insuficiência de pontos de atenção. Por consequência, os hospitais terciários dos grandes centros urbanos, mesmo com níveis adequados de qualidade e de incorporação tecnológica, não conseguem fazer frente a uma demanda massiva, qualitativamente enviesada por motivos de internação sensíveis à atenção primária e por outras causas de internação passíveis de serem resolvidas em outros níveis que não o terciário. As falhas na ordenação do sistema ficam explícitas na regulação insuficiente e/ou errônea dos poucos recursos ambulatoriais e diagnósticos, com extensas e históricas listas de espera e altas taxas de absenteísmo nas vagas ofertadas. ${ }^{11}$ Como resultante, no cenário sanitário tem-se um Sistema Único de Saúde (SUS) fragmentado para enfrentar uma tripla carga de doenças, incrementada pelo envelhecimento populacional. ${ }^{12}$

Para dar suporte ao enfrentamento desse quadro assistencial e sanitário, inicialmente da APS e mais recentemente das RAS, o Programa Telessaúde Brasil Redes é uma iniciativa governamental criada em 2007 e em expansão a partir de então. ${ }^{13}$ Dentro das três ações de telessaúde mais utilizadas no país (teleconsultoria, telediagnóstico e teleducação), a teleconsultoria tem sido vista como uma ótima ferramenta de ajustamento entre pares, além de qualificar e evitar encaminhamentos da APS para outros níveis de atenção. ${ }^{14}$ Por meio de Tecnologias da Informação e Comunicação, como a Internet e o telefone, 
e baseada na melhor evidência possível, a teleconsultoria provê suporte assistencial para profissionais de saúde solicitantes da APS, fornecido por teleconsultores especialistas em APS ou especialistas focais de acordo com a decisão de um profissional de telerregulação. ${ }^{15}$

Programas de saúde podem ser avaliados em relação ao processo (por meio de indicadores de oferta, utilização e cobertura), ao resultado (por meio de indicadores de impacto) ou ambos. Além disso, para se afirmar se os ganhos em processo e/ou resultado foram devidos ao programa, podem ser utilizadas inferências de adequação, plausabilidade e probabilidade. Avaliações de adequação indicam se os objetivos do programa foram alcançados; de plausabilidade, se foram alcançados devido ao programa; e de probabilidade, se o programa foi estaticamente efetivo. Por serem mais simples e menos dispendiosas, avaliações de adequação devem preceder as de plausabilidade e probabilidade, o que é o caso quando se analisa um programa de saúde inovador e em sedimentação, como o Programa Telessaúde Brasil Redes. ${ }^{16}$

Partindo da base legal brasileira e da produção do Núcleo de Telessaúde do Rio Grande do Sul (TelessaúdeRS-UFRGS), um estudo propôs um modelo de processo para teleconsultorias de texto e vídeo, assim como para outras tipologias de ações em telessaúde. ${ }^{17}$ Baseado nesse modelo, numa parceria entre o Ministério da Saúde e a Universidade Federal do Rio Grande do Sul, por meio do Programa de Pós-Graduação em Epidemiologia da Faculdade de Medicina foi produzida a Plataforma Nacional de Telessaúde. Como resultado obteve-se uma solução tecnológica para a telessaúde brasileira, utilizável por qualquer núcleo de telessaúde do país e acessível para profissionais da Atenção Primária à Saúde de toda a rede de atenção à saúde do território nacional, que foi lançada em outubro de 2013 (sob registro INPI № BR 512013 00120), com nova versão em fevereiro de 2015 (em processo de registro).

APlataforma Nacional de Telessaúde foi desenvolvida com uma estrutura de dados robusta e maleável, que permite a inclusão de novos módulos, atores e processos e é compatível com os cadastros nacionais do SUS. Além disso, possui uma entrada de dados simplificada, que facilita o acesso aos serviços, aliada a uma saída de dados complexa, composta por tabelas planas de estrutura e processo, que acumulam todas as variáveis de solicitação, telerregulação, resposta e avaliação das teleconsultorias. Os primeiros módulos dão conta dos fluxos de teleconsultorias assíncronas (solicitante e teleconsultor não estabelecem contato ao mesmo tempo, são usados formulários eletrônicos) e síncronas (solicitante e teleconsultor estabelecem contato simultâneo, são usadas ferramentas de videoconferência). Com base nessa estrutura de dados, este artigo avalia a adequação da oferta e da utilização de teleconsultorias pelos núcleos de telessaúde e usuários que utilizam a Plataforma Nacional de Telessaúde no período de outubro de 2013 a setembro de 2015.

\section{Método}

Estudo descritivo baseado em análise estatística de ações de telessaúde. Foi levantada uma série histórica de 24 meses da produção das ações de teleconsultoria assíncrona e síncrona (texto e vídeo), por todos os núcleos de telessaúde que utilizaram a Plataforma Nacional de Telessaúde a partir do mês de seu lançamento (outubro de 2013) até setembro de 2015.

Para estimar a oferta média, foi considerado como numerador, para cada mês do período, a capacidade (média) de produzir duas teleconsultorias por dia multiplicada pela quantidade de teleconsultores em 
atividade em cada núcleo de telessaúde (uma teleconsultoria a cada duas horas, numa jornada de 20 horas semanais) e como denominador o número de unidades de saúde cadastradas na área de abrangência dos núcleos.

Para avaliação da utilização, foi calculada a média mensal de solicitações por unidade de saúde, considerando como numerador o total mensal de solicitações respondidas por cada núcleo de telessaúde e como denominador o total de unidades de saúde cadastradas (e que, portanto, receberam capacitação no uso da ferramenta) na área de abrangência de cada um desses núcleos.

Para descrição da demanda, foi utilizado o percentual mensal de unidades de saúde ativas e o percentual de usuários ativos em cada categoria profissional (ativos = que realizaram ao menos uma solicitação no período de 24 meses). Também foram descritos os temas mais solicitados, categorizados pela Classificação Internacional de Atenção Primária, versão 2 (CIAP 2) e pela Classificação Internacional de Doenças, versão 10 (CID 10), bem como a distribuição de solicitações por categoria profissional do solicitante e do teleconsultor.

\section{Resultados}

Por meio da Plataforma Nacional de Telessaúde (Tabela 1), foram respondidas 29.575 teleconsultorias (98\% assíncronas) no período de 24 meses, de outubro de 2013 a setembro de 2015 . Essa oferta de serviços foi realizada por 18 núcleos de telessaúde, para 43.421 usuários em 9.801 unidades de saúde cadastradas. Aárea de abrangência dos núcleos de telessaúde incluiu 1.917 municípios de 14 estados das cinco regiões do país. A força de trabalho cadastrada nos núcleos de telessaúde foi de 780 teleconsultores, 132 telerreguladores, sendo que muitos teleconsultores acumulam essas duas funções. Teleconsultores e telerreguladores foram apoiados por um total de 153 monitores.

A Tabela 2 mostra a oferta e a demanda mensal de teleconsultorias no período de estudo, considerando as unidades de saúde contidas na área de abrangência de cada núcleo. Ambas se mantiveram estáveis, com variações entre 0,92 a 2,06 e 0,22 a 1,00 teleconsultorias, respectivamente. O percentual de unidades de saúde ativas (que realizaram ao menos uma solicitação no mês) manteve-se próximo a 0,1\% unidades de saúde. Em função do baixo percentual de unidades ativas, a mediana e a moda da demanda mensal por unidade de saúde foram nulas.

A Tabela 3 mostra as categorias profissionais responsáveis por $5 \%$ ou mais do total de solicitações de teleconsultoria. Todas as outras 41 categorias cadastradas, que acumulam apenas 13,5\% dos usuários, tiveram um percentual abaixo de $1 \%$ do total de solicitações, sendo que a maioria não realizou nenhuma solicitação. Enfermeiros destacaram-se tanto no número absoluto de solicitações quanto no percentual de usuários ativos, embora esse indicador esteja baixo para todas as categorias profissionais, já que foi considerado como ativo aquele solicitante que fez ao menos uma solicitação no período de 24 meses. Como reflexo disso, 86,9\% dos usuários cadastrados não realizou nenhuma solicitação em 24 meses. Em relação aos médicos, esse percentual chega a 90,0\%.

No que diz respeito à profissão dos teleconsultores, a maioria das solicitações de teleconsultoria $(96,0 \%)$ foi respondida por clínicos gerais $(72,5 \%)$, enfermeiros $(19,3 \%)$ e cirurgiões-dentistas $(4,2 \%)$. No cadastro de teleconsultores, essas categorias profissionais apresentam, respectivamente, as frequências de $40,2 \%, 18,7 \%$ e $7,5 \%$. 
Tabela 1. Dados de estrutura e processo de teleconsultorias síncronas e assíncronas dos núcleos de telessaúde que utilizaram a Plataforma Nacional de Telessaúde, no período de outubro de 2013 a setembro de 2015. TelessaúdeRS-UFRGS. Porto Alegre, 2015.

\begin{tabular}{|c|c|c|c|c|c|c|c|c|c|}
\hline Núcleo de telessaúde & UF & $\begin{array}{l}\text { Número de } \\
\text { municípios }\end{array}$ & $\begin{array}{l}\text { Número de } \\
\text { unidades } \\
\text { de saúde }\end{array}$ & $\begin{array}{l}\text { Número de } \\
\text { usuários }\end{array}$ & $\begin{array}{l}\text { Número de } \\
\text { teleconsul- } \\
\text { tores }\end{array}$ & $\begin{array}{c}\text { Número de } \\
\text { regulado- } \\
\text { res* }^{*}\end{array}$ & $\begin{array}{l}\text { Número de } \\
\text { monitores }\end{array}$ & $\begin{array}{l}\text { Período } \\
\text { em meses }\end{array}$ & $\begin{array}{c}\text { Número de } \\
\text { teleconsul- } \\
\text { torias }\end{array}$ \\
\hline ANDRADINA & $\mathrm{SP}$ & 39 & 113 & 894 & 3 & 3 & 0 & 2 & 7 \\
\hline ARAPIRACA & $\mathrm{AL}$ & 39 & 206 & 2.720 & 8 & 8 & 2 & 24 & 602 \\
\hline BACABAL & MA & 11 & 82 & 800 & 4 & 2 & 4 & 13 & 472 \\
\hline BAHIA & $\mathrm{BA}$ & 416 & 3.340 & 12.756 & 40 & 9 & 6 & 24 & 1.127 \\
\hline CEÁRA & CE & 174 & 1.113 & 3.979 & 81 & 6 & 9 & 18 & 249 \\
\hline CRISTALINA & GO & 7 & 143 & 453 & 8 & 8 & 2 & 21 & 202 \\
\hline GARÇA & $\mathrm{SP}$ & 27 & 111 & 572 & 6 & 6 & 0 & 6 & 13 \\
\hline HUUFMA & MA & 45 & 255 & 2.402 & 27 & 4 & 6 & 14 & 3.666 \\
\hline JOÃO PESSOA & PB & 6 & 84 & 1.622 & 13 & 4 & 5 & 10 & 456 \\
\hline NÚCLEO DE TELESSAÚDE DO IMIP & PE & 62 & 157 & 444 & 96 & 14 & 10 & 10 & 77 \\
\hline $\begin{array}{l}\text { NÚCLEO TÉCNICO-CIENTíFICO } \\
\text { DE TELESSAÚDE DO ESTADO DO } \\
\text { AMAZONAS }\end{array}$ & AM & 35 & 90 & 220 & 19 & 4 & 2 & 3 & 20 \\
\hline $\begin{array}{l}\text { NÚCLEO TÉCNICO-CIENTÍFICO DE } \\
\text { TELESSAÚDE DE MATO GROSSO }\end{array}$ & MT & 140 & 814 & 3.650 & 149 & 7 & 15 & 14 & 710 \\
\hline $\begin{array}{l}\text { NÚCLEO TÉCNICO-CIENTÍFICO DE } \\
\text { TELESSAÚDE PARÁ }\end{array}$ & PA & 82 & 265 & 365 & 18 & 1 & 12 & 4 & 8 \\
\hline PARANÁ & PR & 272 & 914 & 3.604 & 41 & 18 & 4 & 22 & 14.808 \\
\hline PETROLNA & PE & 22 & 265 & 931 & 22 & 5 & 1 & 24 & 743 \\
\hline SANTA INÊS & MA & 11 & 97 & 903 & 6 & 2 & 6 & 2 & 6 \\
\hline SERGIPE & SE & 75 & 269 & 2.987 & 11 & 10 & 15 & 23 & 656 \\
\hline TELESSAÚDERS & $\mathrm{RS}$ & 454 & 1.483 & 4.119 & 228 & 21 & 54 & 24 & 5.753 \\
\hline Totais & & 1.917 & 9.801 & 43.421 & 780 & 132 & 153 & & 29.575 \\
\hline
\end{tabular}

* Em geral, teleconsultores acumulam a função de telerreguladores.

A Tabela 4 mostra que foram realizadas solicitações de teleconsultoria com temas distribuídos em todos os capítulos da CIAP 2. Dentro do capítulo A - Geral e inespecífico houve 4.589 (15,6\%) solicitações classificadas sob o título Eletrocardiograma e dentro do capítulo N - Sistema nervoso houve 2.660 (9,0\%) classificadas como Cefaleia e 1.285 (4,3\%) como Epilepsia. Embora apenas 1.611 solicitações tenham sido classificadas por meio da CID 10, já que esta é opcional e complementar à CIAP 2, também houve solicitações em todos os capítulos da CID 10.

Como a avaliação dos serviços é opcional, $50,2 \%$ das solicitações de teleconsultorias foram avaliadas, com $95,6 \%$ dos solicitantes satisfeitos com o serviço e $88,4 \%$ declarando que tiveram suas dúvidas totalmente respondidas. Também por meio da avaliação verificou-se que em 4.288 solicitações realizadas por médicos havia intenção de encaminhamento em 2.497 (58,2\%) dos casos e, desses últimos, houve evitação do encaminhamento em $34,8 \%$ dos casos.

\section{Discussão}

Resumo dos principais achados do estudo

A estrutura implantada é compatível com o processo e capaz de atingir as metas do Programa Telessaúde Brasil Redes. Conforme o modelo de avaliação proposto, a capacidade instalada de oferta 
Tabela 2. Evolução da oferta e da demanda de teleconsultorias síncronas e assíncronas na Plataforma Nacional de Telessaúde, no período de outubro de 2013 a setembro de 2015. TelessaúdeRS-UFRGS. Porto Alegre, 2015.

\begin{tabular}{|c|c|c|c|c|c|c|c|c|}
\hline $\begin{array}{l}\text { Período de } \\
\text { telessaúde } \\
\text { em atividade }\end{array}$ & $\begin{array}{l}\text { Número de } \\
\text { núcleos }\end{array}$ & $\begin{array}{l}\text { Número de } \\
\text { unidades de } \\
\text { saúde ca- } \\
\text { dastradas }\end{array}$ & $\begin{array}{c}\text { \% de unida- } \\
\text { des de saú- } \\
\text { de ativas }\end{array}$ & $\begin{array}{l}\text { Número de } \\
\text { solicitações }\end{array}$ & $\begin{array}{l}\text { Média mensal } \\
\text { de demanda de } \\
\text { solicitações por } \\
\text { unidade de saú- } \\
\text { de cadastrada }\end{array}$ & $\begin{array}{l}\text { Número de } \\
\text { teleconsul- } \\
\text { tores em } \\
\text { atividade }\end{array}$ & $\begin{array}{l}\text { Média mensal de } \\
\text { oferta de tele- } \\
\text { consultorias por } \\
\text { unidade de saú- } \\
\text { de cadastrada }\end{array}$ & $\begin{array}{c}\text { Versão } \\
\text { Plataforma }\end{array}$ \\
\hline out/13 & 5 & 3.404 & 0,15 & 929 & 0,38 & 42 & 1,45 & 1.9 \\
\hline nov/13 & 6 & 3.404 & 0,09 & 586 & 0,23 & 46 & 0,96 & 1.9 \\
\hline $\operatorname{dez} / 13$ & 6 & 3.404 & 0,07 & 448 & 0,22 & 44 & 0,95 & 1.9 \\
\hline $\mathrm{jan} / 14$ & 7 & 3.426 & 0,09 & 578 & 0,29 & 48 & 1,10 & 1.9 \\
\hline fev/14 & 7 & 3.426 & 0,09 & 666 & 0,53 & 53 & 1,41 & 1.9 \\
\hline $\mathrm{mar} / 14$ & 7 & 3.426 & 0,08 & 544 & 0,28 & 52 & 2,06 & 1.9 \\
\hline $\mathrm{abr} / 14$ & 8 & 3.426 & 0,09 & 831 & 0,27 & 56 & 1,34 & 1.9 \\
\hline mai/14 & 8 & 3.426 & 0,08 & 710 & 0,26 & 53 & 1,18 & 1.9 \\
\hline jun/14 & 6 & 3.426 & 0,07 & 692 & 0,30 & 53 & 1,03 & 1.9 \\
\hline $\mathrm{jul} / 14$ & 7 & 3.426 & 0,07 & 981 & 0,47 & 50 & 1,37 & 1.9 \\
\hline ago/14 & 10 & 3.426 & 0,09 & 1.265 & 0,53 & 54 & 1,15 & 1.9 \\
\hline set/14 & 10 & 3.426 & 0,08 & 1.508 & 0,98 & 61 & 1,64 & 1.9 \\
\hline out/14 & 10 & 3.426 & 0,09 & 1.816 & 1,00 & 60 & 1,55 & 1.9 \\
\hline nov/14 & 9 & 3.426 & 0,07 & 1.142 & 0,67 & 54 & 1,33 & 1.9 \\
\hline $\mathrm{dez} / 14$ & 12 & 3.426 & 0,07 & 1.084 & 0,46 & 63 & 1,45 & 1.9 \\
\hline $\mathrm{jan} / 15$ & 10 & 3.440 & 0,04 & 608 & 0,36 & 50 & 1,60 & 1.9 \\
\hline $\mathrm{fev} / 15$ & 11 & 3.691 & 0,06 & 982 & 0,42 & 53 & 1,52 & 2.0 \\
\hline mar/15 & 12 & 4.025 & 0,07 & 1.379 & 0,63 & 62 & 1,55 & 2.0 \\
\hline$a b r / 15$ & 14 & 4.551 & 0,07 & 1.297 & 0,51 & 84 & 1,97 & 2.0 \\
\hline mai/15 & 14 & 9.212 & 0,04 & 1.863 & 0,37 & 96 & 0,92 & 2.0 \\
\hline jun/15 & 14 & 9.338 & 0,04 & 1.932 & 0,41 & 98 & 1,55 & 2.0 \\
\hline $\mathrm{jul} / 15$ & 15 & 9.491 & 0,05 & 2.280 & 0,46 & 118 & 1,47 & 2.0 \\
\hline ago/15 & 17 & 9.647 & 0,06 & 2.721 & 0,54 & 147 & 1,23 & 2.0 \\
\hline set/15 & 17 & 9.801 & 0,05 & 2.733 & 0,49 & 157 & 1,06 & 2.0 \\
\hline Total & & & & 29.575 & & & & \\
\hline
\end{tabular}

Tabela 3. Categorias profissionais responsáveis por $5 \%$ ou mais das solicitações de teleconsultorias síncronas e assíncronas da Plataforma Nacional de Telessaúde no período de outubro de 2013 a setembro de 2015. TelessaúdeRS-UFRGS. Porto Alegre, 2015.

\begin{tabular}{|c|c|c|c|c|c|c|}
\hline Profissão de solicitante & $\begin{array}{c}\text { Número de } \\
\text { usuários } \\
\text { cadastrados }\end{array}$ & $\begin{array}{l}\text { \% do total } \\
\text { de usuários } \\
\text { cadastrados }\end{array}$ & $\begin{array}{l}\text { Número de } \\
\text { usuários } \\
\text { ativos }\end{array}$ & $\begin{array}{c}\% \text { de usuários } \\
\text { ativos }\end{array}$ & $\begin{array}{l}\text { Números de } \\
\text { solicitações }\end{array}$ & $\begin{array}{c}\% \text { do total de } \\
\text { solicitações }\end{array}$ \\
\hline ENFERMEIRO(A) & 11.083 & 25,3 & 2.132 & 19,2 & 16.011 & 54,1 \\
\hline MÉDICO(A) CLÍNICO(A) & 8.509 & 19,4 & 850 & 10,0 & 4.244 & 14,3 \\
\hline AGENTE COMUNITÁRIO(A) DE SAÚDE & 11.452 & 26,1 & 1.331 & 11,6 & 3.609 & 12,2 \\
\hline TÉCNICO(A) EM ENFERMAGEM & 3.072 & 7,0 & 304 & 9,9 & 2.429 & 8,2 \\
\hline CIRURGIÃO(A)-DENTISTA & 3.788 & 8,6 & 404 & 10,7 & 1.520 & 5,1 \\
\hline$\ldots$ & $\ldots$ & $\ldots$ & $\ldots$ & $\ldots$ & $\ldots$ & $\ldots$ \\
\hline Totais & 43.421 & 100,0 & 5.667 & 13,1 & 29.575 & 100,0 \\
\hline
\end{tabular}


Tabela 4. Número de solicitações de teleconsultorias síncronas e assíncronas categorizadas pela Classificação Internacional de Atenção Primária - versão 2 na Plataforma Nacional de Telessaúde no período de outubro de 2013 a setembro de 2015. TelessaúdeRS-UFRGS. Porto Alegre, 2015.

\begin{tabular}{lcc}
\hline Capítulos & Número de solicitações* & $\%$ do total de solicitações \\
\hline A - Geral e inespecífico & 12.772 & $43,3 \%$ \\
N - Sistema nervoso & 7.159 & $24,2 \% \%$ \\
P - Psicológico & 1.627 & $5,5 \%$ \\
K - Aparelho circulatório & 1.415 & $4,8 \%$ \\
W - Gravidez e planeamento familiar & 1.368 & $4,6 \%$ \\
D - Aparelho digestivo & 1.115 & $3,8 \%$ \\
X - Aparelho genital feminino (incluíndo mama) & 899 & $3,0 \%$ \\
S - Pele & 814 & $2,8 \%$ \\
T - Endócrino, metabólico e nutricional & 657 & $2,2 \%$ \\
L - Sistema musculo-esquelético & 388 & $1,3 \%$ \\
B - Sangue, órgãos hematopoiéticos e linfáticos & 370 & $1,3 \%$ \\
R - Aparelho respiratório & 261 & $0,9 \%$ \\
Y - Aparelho genital masculino & 193 & $0,7 \%$ \\
F - Olhos & 182 & $0,6 \%$ \\
U - Aparelho urinário & 175 & $0,6 \%$ \\
H - Ouvidos & 66 & $0,2 \%$ \\
Z - Problemas sociais & 61 & $0,2 \%$ \\
\hline Total Geral & 29.522 & $100,0 \%$ \\
\hline
\end{tabular}

* Um conjunto de 53 solicitações não foi classificado por uma falha temporária na validação de entrada de dados.

mensal média de teleconsultorias por unidade de saúde, atingida pelos núcleos de telessaúde, alcançou a meta de oferta de uma a três solicitações mensais estabelecida pelo Ministério da Saúde. ${ }^{18}$ Porém, no contraponto de um grande leque de temas explorado nas teleconsultorias, existe uma baixa demanda dos profissionais da APS por essa tipologia de serviços de telessaúde. A grande quantidade de solicitações em temas muito específicos como eletrocardiograma, cefaleia e epilepsia pode significar o uso adaptado do fluxo de teleconsultorias síncronas e assíncronas para experiências em telediagnóstico e apoio ao complexo regulador ambulatorial.

\section{Fortalezas e limitações do estudo}

O presente estudo fornece uma avaliação da oferta e da demanda dos serviços de teleconsultorias síncronas e assíncronas (texto e vídeo) em 18 (43\%) dos núcleos de telessaúde em operação no país, já que, conforme dados do Programa Telessaúde Brasil Redes, existiam 42 núcleos em operação em agosto de 2015. ${ }^{19}$ Porém, ao se avaliar uma possível capacidade instalada ociosa, decorrente da baixa utilização, é preciso considerar que a justificativa de investimento público em telessaúde no Brasil parte de um contexto de expansão e inovação. Além disso, os núcleos de telessaúde também realizam atividades de telediagnóstico e de teleducação, bem como outras modalidades de teleconsultoria (por exemplo.: via telefone) não incluídas dentro da análise deste artigo. A baixa utilização geral e em especial a baixa utilização por médicos inviabiliza a utilização de indicadores de resultado, como a evitação de encaminhamentos. 


\section{Comparação com a literatura já existente}

No que diz respeito à utilização, dois dos três principais fatores associados à usabilidade de ferramentas de telessaúde - simplicidade e treinamento suficiente - são cumpridos pela Plataforma Nacional de Telessaúde. Mesmo assim, a média mensal de demanda espontânea de solicitações por unidade de saúde ficou bem abaixo dos valores entre 1,5 e 1,9 solicitações mensais por unidade encontrados em experiências nacionais e internacionais. ${ }^{20,21}$ Apesar da baixa utilização ser um problema, ela é uma realidade da demanda espontânea nacional e internacional.

Isso é reforçado por um estudo recente (2015), ao sugerir que clínicos preferem encaminhar pacientes para consultas em outros níveis de atenção do que solicitar suporte assistencial por telessaúde para resolver os casos localmente e com qualidade na APS. ${ }^{17}$ Outro estudo já havia demonstrado um percentual de $30 \%$ de usuários médicos cadastrados que não utilizavam um serviço de teleconsultorias. ${ }^{22} \mathrm{~A}$ disposição em usar serviços por médicos foi denominada como aceitação clínica e é considerada um fator decisivo para a sustentação de novos serviços. ${ }^{23}$ Tanto o problema da baixa utilização quanto da baixa aceitação clínica, bem como do aperfeiçoamento profissional, são de natureza multifatorial e requerem ações multifacetadas no seu enfrentamento. ${ }^{24}$

\section{Implicações para a pesquisa na área e/ou para a prática dos profissionais}

a) Ganho de escala e integração horizontal: A tipologia de solicitação de teleconsultorias via Plataforma Nacional de Telessaúde exige uma infraestrutura mínima de conectividade e equipamentos de interação a distância. Das 38.812 unidades básicas de saúde avaliadas pelo Ministério da Saúde, 35\% referiram essas condições. ${ }^{25}$ Muitas dessas unidades estão fora das áreas de abrangências dos núcleos de telessaúde, que normalmente são limitadas por fronteiras municipais ou estaduais. A limitação de áreas de abrangência por fronteiras geográficas contraria as possibilidades de economia de escala e de integração horizontal e é incoerente com a premissa básica da telessaúde, que diz respeito a minimizar as limitações impostas pela distância.

Em setembro de 2015, a Plataforma Nacional de Telessaúde acumulou 780 teleconsultores, porém apenas a quinta parte desta capacidade instalada (157 teleconsultores) realizou produção de teleconsultorias (Tabelas 1 e 2). Caso todos tivessem uma produção plena de 40 teleconsultorias por mês, sem considerar a limitação das áreas de abrangência, seria possível produzir 31.200 teleconsultorias, o que equivaleria a 11 vezes a produção do mês de setembro de 2015 e a 3,2 teleconsultorias mensais por unidade de saúde cadastrada.

Considerando a atual demanda espontânea, essa capacidade instalada permitiria atender a todas as unidades de APS do país. Se por um lado é possível ter ganho de escala, por outro lado, é necessário concentrar a aplicação de recursos. Em tempos de contingenciamentos orçamentários para a saúde, não há justificativa para a existência de 42 núcleos de telessaúde. Um ou dois núcleos por região do país, todos integrados horizontalmente, seriam mais do que suficientes para coordenar o atual grupo de teleconsultores, sendo possível manter a dispersão geográfica dos teleconsultores.

b) Telerregulação e auditoria: a regulação em telessaúde é um ponto necessário e fulcral em função da evidência de iatrogenia gerada pela solicitação desregrada e/ou desnecessária de procedimentos, sejam 
eles de teleconsultoria ou de telediagnóstico. ${ }^{26,27}$ Intervenções que buscam reestruturar práticas clínicas, associando-as com grupos de referência (o que inclui auditoria, retroalimentação e lembretes) são mais propensas para levar a mudanças de comportamento.

Da mesma forma, existe demonstração de inefetividade, para aperfeiçoamento clínico, da disseminação de informação não solicitada, como palestras, aulas expositivas e diretrizes clínicas, indicando melhores resultados com intervenções multifacetadas, como visitas educacionais e auditoria com retroalimentação. . $^{24,28}$ Portanto, a telerregulação potencializa a educação permanente ao conectar o solicitante ao teleconsultor ideal para a discussão do caso.

A telerregulação também é uma ferramenta de integração horizontal no momento em que telerreguladores das diversas regiões do país podem colaborar para distribuir solicitações de acordo com protocolos baseados em evidências. Da mesma forma, a auditoria por pares, além de incidir na qualidade do serviço prestado, pode potencializar a telerregulação. ${ }^{12}$

c) Novas tipologias de ações de telessaúde: é preciso repensar o modelo de oferta de teleconsultorias por meio da web, em que o solicitante tem que reservar tempo para acessar um terminal por meio de acesso registrado. Nesse sentido, pelas estimativas da Pesquisa Nacional por Amostra de Domicílios (PNAD) em 2013, o acesso exclusivo por computador vem sofrendo retração, indicando o crescimento de outras formas de acesso, como o telefone celular e tablet (responsáveis por 4,1\% dos acessos).

No Brasil, $75,2 \%$ da população de 10 anos ou mais de idade possui telefone celular (aumento de 131,4\% em relação a 2005). ${ }^{29}$ É necessário o uso de múltiplas tipologias de oferta de serviços de telessaúde, que aliem o atual uso da internet, telefone tradicional e dispositivos móveis inteligentes (smartphones). Com isso, será possível oferecer serviços com maior aceitação para profissionais de saúde, gestores e mesmo pacientes, mesclando todas as possibilidades de ações de telessaúde.

A aceitação clínica deixa de ser uma barreira quando os novos serviços se inserem na prática diária. Tipologias de ações em telessaúde que partam de outras necessidades, que não apenas a demanda espontânea por profissionais de saúde, como a pressão assistencial exercida pelas necessidades dos pacientes por consultas e procedimentos ambulatoriais, são um exemplo disso. Iniciativas de apoio ao complexo regulador ambulatorial produzem sinergia entre diversas tipologias de ações de telessaúde, como teleconsultorias via web e via telefone e telediagnóstico. ${ }^{17}$

Finalmente, embora a teleconsulta (interação entre profissional de saúde e paciente) ainda não seja uma realidade nacional (não é permitida pelo Conselho Federal de Medicina, apesar da dimensão continental do país e das grandes iniquidades na distribuição dos recursos humanos em saúde, mesmo com a telessaúde já tendo sido regulamentada pelo Conselho Federal de Medicina ainda em 2002), ${ }^{30,31}$ são amplas as possibilidades de uso da atual capacidade instalada para orientação e educação em saúde, bem como no enfrentamento de endemias, epidemias e outros eventos populacionais.

\section{Conclusões}

O baixo uso e os percentuais acima de $80 \%$ de solicitantes sem nenhuma utilização reforçam a necessidade de avaliação do modelo de oferta de teleconsultorias, pois aguardar por demanda espontânea não parece ser o caminho. Apesar da oferta adequada em relação às metas do programa, a demanda 
é muito baixa, gerando capacidade instalada ociosa de um grupo de teleconsultores com capacidade de resposta para um amplo leque de temas. Levanta-se a necessidade de ganho de escala, integração horizontal e fortalecimento da telerregulação e da auditoria das ações de telessaúde, com centralização de recursos e redução do número de núcleos de telessaúde. Além disso, é necessário investir em novas tipologias sinérgicas e sistêmicas de oferta de ações de telessaúde, como o apoio ao complexo regulador ambulatorial e à orientação da população.

\section{Agradecimentos}

O presente trabalho não seria possível sem o apoio técnico, político e financeiro do Ministério da Saúde, da Secretaria Estadual de Saúde do Rio Grande do Sul e da Universidade Federal do Rio Grande do Sul, com participação da Faculdade de Medicina e do Programa de Pós-Graduação em Epidemiologia. Também foi importante o respaldo técnico e científico da equipe do TelessaúdeRS-UFRGS.

\section{Referências}

1. Organización Panamericana de la Salud. Informe Dawson sobre el futuro de los servicios médicos y afines, 1920. Washington: OPAS; 1964. $38 \mathrm{p}$.

2. Starfield B. Atenção primária: equilíbrio entre necessidades de saúde, serviços e tecnologia. Brasília/UNESCO: Ministério da Saúde; 2002.

3. Brasil. Decreto n. 7.508, 28 de junho de 2011. Regulamenta a Lei no 8.080, de 19 de setembro de 1990, para dispor sobre a organização do Sistema Único de Saúde - SUS, o planejamento da saúde, a assistência à saúde e a articulação interfederativa, e dá outras providências Brasília: Diário Oficial da União; 2011;29.

4. Brasil. Ministério da Saúde. Histórico de cobertura da Saúde da Família. Brasília: Ministério da Saúde; 2015.

5. Harzheim E, ed. Reforma da atenção primária à saúde na cidade do Rio de Janeiro: avaliação dos primeiros três anos de clínicas da família. Porto Alegre: OPAS; 2013.

6. Macinko J, de Oliveira VB, Turci MA, Guanais FC, Bonolo PF, Lima-Costa MF. The influence of primary care and hospital supply on ambulatory care-sensitive hospitalizations among adults in Brazil, 1999-2007. Am J Public Health. 2011;101(10):1963-70. DOI: http:// dx.doi.org/10.2105/AJPH.2010.198887

7. Gonçalves MR, Hauser L, Prestes IV, Schmidt MI, Duncan BB, Harzheim E. Primary health care quality and hospitalizations for ambulatory care sensitive conditions in the public health system in Porto Alegre, Brazil. Fam Pract. 2016;33(3):238-42. DOI: http://dx.doi.org/10.1093/ fampra/cmv051

8. Chomatas E, Vigo A, Marty I, Hauser L, Harzheim E. Avaliação da presença e extensão dos atributos da atenção primária em Curitiba. Rev Bras Med Fam Comunidade. 2013;8(29):294-303. DOI: http://dx.doi.org/10.5712/rbmfc8(29)828

9. Castro RCL, Knauth DR, Harzheim E, Hauser L, Duncan BB. Avaliação da qualidade da atenção primária pelos profissionais de saúde: comparação entre diferentes tipos de serviços. Cad Saúde Pública. 2012;28(9):1772-84. DOI: http://dx.doi.org/10.1590/S0102311X2012000900015

10. Facchini LA, Piccini RX, Tomasi E, Thumé E, Silveira DS, Siqueira FV, et al. Performance of the PSF in the Brazilian South and Northeast: institutional and epidemiological Assessment of Primary Health Care. Ciênc Saúde Coletiva. 2006;11(3):669-81. DOI: http://dx.doi. org/10.1590/S1413-81232006000300015

11. Harzheim E. Panorama tecnológico da área de telemedicina do complexo da saúde. Brasília: Agência Brasileira de Desenvolvimento Industrial; 2015.

12. Mendes EV. As redes de atenção à saúde. Brasília: Organização Pan-Americana da Saúde; 2011.

13. Harzheim E, Gonçalves MR, Umpierre RN, da Silva Siqueira AC, Katz N, Agostinho MR, et al. Telehealth in Rio Grande do Sul, Brazil: Bridging the Gaps. Telemed J E Health. 2016;22(11):938-44. DOI: http://dx.doi.org/10.1089/tmj.2015.0210

14. Mendes EV. A construção social da atenção primária à saúde. Brasília: Conselho Nacional de Secretários de Saúde; 2015. 
15. Brasil. Ministério da Saúde. Manual de Telessaúde para Atenção Básica/Atenção Primária à Saúde. Brasília: Ministério da Saúde; 2012.

16. Habicht JP, Victora CG, Vaughan JP. Evaluation designs for adequacy, plausibility and probability of public health programme performance and impact. Int J Epidemiol. 1999;28(1):10-8. DOI: http://dx.doi.org/10.1093/ije/28.1.10

17. Schmitz CAA. Telessaúde como suporte assistencial para a Atenção Primária à Saúde no Brasil [tese]. Porto Alegre: Universidade Federal do Rio Grande do Sul; 2015.

18. Brasil. Ministério da Saúde. Portaria no 2.860, de 28 de dezembro de 2014. Define os valores do incentivo financeiro de custeio mensal destinado aos Núcleos de Telessaúde do Programa Nacional Telessaúde Brasil Redes na Atenção Básica de que trata a Portaria no 2.859/GM/MS, de 29 de dezembro de 2014. Brasília: Diário Oficial da União; 2014.

19. Brasil. Ministério da Saúde. Secretaria de Gestão do Trabalho e da Educação na Saúde. Apresentação da produção de atividades dos Núcleos de Telessaúde. Brasília: Ministério da Saúde; 2015.

20. Alkmim MBM. Fatores associados à utilização de sistema de teleconsultoria na atenção primária de municípios remotos de Minas Gerais [dissertação]. Belo Horizonte: Universidade Federal de Minas Gerais; 2010.

21. Mars M, Scott R. Telemedicine service use: a new metric. J Med Internet Res. 2012;14(6):e178. DOI: http://dx.doi.org/10.2196/jmir.1938

22. Castro Filho ED. Telessaúde no apoio a médicos de atenção primária [tese]. Porto Alegre: Faculdade de Medicina, Universidade Federal do Rio Grande do Sul; 2011.

23. Wade VA, Eliott JA, Hiller JE. Clinician acceptance is the key factor for sustainable telehealth services. Qual Health Res. 2014;24(5):68294. DOI: http://dx.doi.org/10.1177/1049732314528809

24. Oxman AD, Thomson MA, Davis DA, Haynes RB. No magic bullets: a systematic review of 102 trials of interventions to improve professional practice. CMAJ. 1995;153(10):1423-31.

25. Brasil. Ministério da Saúde. Departamento de Atenção Básica. Microdados da avaliação externa. Brasília: Ministério da Saúde; 2015. [acesso 2017 Jul 12]. Disponível em: http://dab.saude.gov.br/portaldab/ape_pmaq.php?conteudo=microdados

26. Croswell JM, Kramer BS, Kreimer AR, Prorok PC, Xu JL, Baker SG, et al. Cumulative incidence of false-positive results in repeated, multimodal cancer screening. Ann Fam Med. 2009;7(3):212-22. DOI: http://dx.doi.org/10.1370/afm.942

27. Jamoulle M, Roland M. Champs d'action, gestion de l'information et formes de prévention clinique en médecine générale et de famille. Santé Conjuguée. 2005;33(7):71-7.

28. Johnson MJ, May CR. Promoting professional behaviour change in healthcare: what interventions work, and why? A theory-led overview of systematic reviews. BMJ Open. 2015;5(9):e008592. DOI: http://dx.doi.org/10.1136/bmjopen-2015-008592

29. Brasil. Ministro do Planejamento, Orçamento e Gestão. Instituto Brasileiro de Geografia e Estatística - IBGE. Pesquisa nacional por amostra de domicílios: acesso à internet e à televisão e posse de telefone móvel celular para uso pessoal. 2014. Rio de Janeiro: IBGE; 2014.

30. Brasil. Conselho Federal de Medicina. Código de Ética Médica. Brasília: CFM; 2009. [acesso 2017 Jul 12]. Disponível em: http://portal. cfm.org.br/index.php?option=com_content\&view=category\&id=9\&ltemid=122

31. Brasil. Conselho Federal de Medicina. Resolução n. 1.643, de 7 de agosto de 2002. Define e disciplina a prestação de serviços através da Telemedicina. Brasília: Diário Oficial da União; 2002. [acesso 2017 Jul 12]. Disponível em: http://www.portalmedico.org.br/resolucoes/ CFM/2002/1643_2002.pdf

a Universidade Federal do Rio Grande do Sul (UFRGS); TelessaúdeRS-UFRGS. Porto Alegre, RS, Brasil. carlos.aita @telessauders.ufrgs.br (Autor correspondente)

b Universidade Federal do Rio Grande do Sul (UFRGS); Secretaria Municipal de Saúde. Porto Alegre, RS, Brasil. erno.harzheim @ telessauders.ufrgs.br 